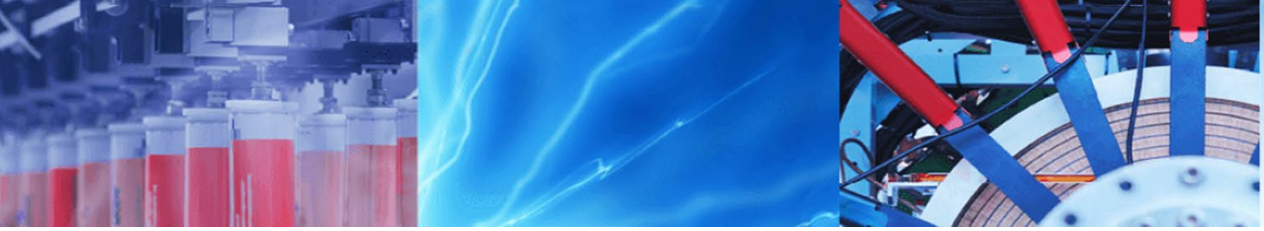

Research Article

\title{
Artificial nacre-like layer using layer coating with bioinspired mesolayer insertions
}

\author{
Pei-Hung Kuo ${ }^{1} \cdot$ Ping-Wen Chen ${ }^{1} \cdot$ Jenq-Gong Duh $^{1}$ (I)
}

Received: 6 September 2019 / Accepted: 11 December 2019 / Published online: 19 December 2019

(c) Springer Nature Switzerland AG 2019

\begin{abstract}
The fabrication of coatings using conventional methods [e.g., physical vapor deposition (PVD) and/or pulsed laser deposition (PLD)] usually creates clear interfaces between the edges. It is challenging to increase the toughness by adding soft materials into hard materials to reinforce the structures using architectural design. In this study, we determined that by adding soft materials into hard materials, we can simulate nacre, which results in stronger coatings. However, the compatibility of materials needs to be considered (i.e., the bonding of organic/inorganic components to each other). Titanium dioxide $\left(\mathrm{TiO}_{2}\right)$, an inorganic material, was chosen as the hard part, and polyimide (PI), an organic material, was chosen as the soft part. When we fused these two materials together to form a layer-by-layer coating, the bonding between them was not good, which degraded the mechanical performance. Nevertheless, we revaluated the differences between artificial and natural coatings. The layers in nacre smoothly change from chitins to proteins or from proteins to chitins owing to the seasonal environmental temperature changes. Inspired by this concept and by simulating nature, we created mesolayers to adhere two different materials and to enhance the coatings that are made entirely of inorganic or organic materials to create new composites using the PVD/PLD method. The mesolayers mixed with two materials at proper ratios were inserted into the organic/inorganic $\left(\mathrm{PI} / \mathrm{TiO}_{2}\right)$ interfaces as bridges to cross-link the materials and dramatically improve the mechanical performance, which is similar to the structure of natural nacre.
\end{abstract}

Keywords Mesolayer · Bioinspired PVD·PLD · Multilayers · Coating

\section{Introduction}

Nature is remarkably adept at using brittle ceramic-like materials as scaffolds for structural applications; the produced materials exhibit excellent strength, toughness, and wear resistance. These characteristics are achieved because of the scaffold structure and soft materials that infiltrate into spaces between the scaffolds to form hierarchical structures. The successful examples include sea shells, pearls, and abalones [1-3]. These materials inspired a new field of composite materials that simulate the abovementioned structures to provide additional bonding and resist the initiation and propagation of cracks [3].
Bioinspired strong materials have been developed using several types of highly technological methods, which reinforce the mechanical performance of these materials. Currently, scientists simulate nature to identify approaches that are required to create functional materials for modern applications [4-15]. Furthermore, structural stability has been enhanced to surpass that of the original materials. Specifically, architectural design is used to manipulate adhesion between layer-by-layer ( $L B L$ ) interfaces using chemical bath deposition (CBD) [4-9], freeze casting (ice-templating) [10, 13], coextrusion [14] approaches to synthesize periodic coatings, and the physical vapor deposition (PVD)/pulsed laser

$\triangle$ Jenq-Gong Duh, jgd@mx.nthu.edu.tw |'Department of Materials Science and Engineering, National Tsing Hua University, No. 101, Section 2, Kuang-Fu Road, Hsinchu 30013, Taiwan. 
deposition (PLD) method [15] is used to form LBL/lamellar structures in a high vacuum system. Using CBD and freeze casting methods, it is possible to create a "brick and mortar" structure that is very similar to that of natural nacre. The PVD/PLD method can only create an LBL structure, and it is a nearly pure physical process without sufficient chemical reactions to create bonding; chemical reactions are possible only in a short range close to the interface. However, the deposition can be well controlled and produce structures with structures that are $1000 \mathrm{~nm}$ thick. Yang et al. have successfully deposited zirconia (an inorganic part) by reactive RF sputtering and polyimide (an organic part) by a pulsed laser deposition system. A multilayered structural hybrid coating was achieved; its fracture toughness exceeded that of the original materials, which implies that the new composites could absorb more energy [15].

In this study, owing to their thermal and chemical stabilities and non-toxic characteristics, titania (a cheap, chemically stable, and widely used material) was chosen as an inorganic part, and a polyimide (PI) nanofilm was used as an organic part to construct nacre-like LBL coatings $[16,17]$. However, the addition of $\mathrm{TiO}_{2}$ may cause reduced elongation of the nanocomposite film, which acts as a non-reactive, non-reinforcing filler if there are no bonding sites between $\mathrm{PI} / \mathrm{TiO}_{2}$ [17]. Previous studies evaluated the compatibility of the physical processes to make sure that complete infiltration without unexpected effects occurred; these studies applied additional post-treatments (e.g., quenching or sintering) to increase bonding $[13,14]$.

To overcome the disadvantages from the poor compatibility of $\mathrm{Pl} / \mathrm{TiO}_{2}$, we implemented two improvements. The first improvement is to increase the repetition of coatings. According to the research by Burghard et al. [6, 7], the bonding between the interface of $\mathrm{PE} / \mathrm{TiO}_{2}$ (organic/ inorganic) is needed to reduce the thickness of the organic layer to allow partial interpenetration to form regions with a mixed composition that are promoted by electrostatic attractions. The second improvement is to create a mixed layer with its organic/inorganic part and insert it into the interface between these two materials without a reliable bonding. By observing the growth of nacre, it was determined that the growth of chitins or proteins does not considerably differ from one to another, and a smooth crossover occurs to form a transition area called mesolayer; this process follows the seasonal changes in temperature. [18-22].

Based on these two concepts, a mixed organic/inorganic mesolayer with a suitable ratio has been introduced to the interface between $\mathrm{PI} / \mathrm{TiO}_{2}$ to improve the adhesion. The thickness of $\mathrm{PI} / \mathrm{TiO}_{2}$ is reduced to induce higher interpenetration in the PI films. More repetitions can be achieved to enhance the compressive stored energy.

\section{Materials and methods}

Pre-cleaned p-type silicon wafers with a $\langle 100\rangle$ orientation were ultrasonically cleaned in DI water, rinsed with a $95 \%$ alcohol, and dried. Subsequent to this procedure, the substrates were ready for deposition. A pure titanium target $(99.99 \%$, Ultimate Materials Technology Co., Ltd., Hsinchu, Taiwan) with a diameter of $5.08 \mathrm{~cm}$ (2 inches) was utilized to deposit a titania film by reactive magnetic sputtering in an argon and oxygen atmosphere. In this study, a polyimide (PI) target was used to deposit polymer interlayers. A polyimide bar (Ultimate Materials Technology Co., Ltd., Hsinchu, Taiwan) $[30 \mathrm{~cm}$ long with a diameter of $2.54 \mathrm{~cm}$ (1 inch)] was evenly sliced into 10 polyimide targets with thicknesses of $3 \mathrm{~cm}$. The exposed surface of the target was flattened and polished by sandpapers and alumina powders.

The hybrid PVD system, which included sputtering and PLD, was designed and integrated in the same vacuum chamber. Two coating systems can be operated within one vacuum chamber sequentially or cooperatively using pre-determined procedures.

The substrate was attached to the substrate holder. The holder is continuously rotated during the sputter and/or PLD processes. In addition, during the PLD procedure, the PI target moved horizontally back and forth to allow the laser spot to irradiate the entire target to minimize the formation of trenches owing to the laser etching phenomenon and to ensure a uniformly vaporized organic film deposited onto the surface of the substrate. The organic film was deposited by the Nd:YAG laser source with built-in harmonics (LS-2137U, Lotis TII Corporation, USA). The wavelength of the UV-pulsed laser was $266 \mathrm{~nm}$, and the energy was set to $30 \mathrm{~mJ}$. The argon atmosphere was introduced into the chamber during the PLD process to induce localized plasma to increase the surface roughness of polymer films. $\mathrm{TiO}_{2} / \mathrm{PI}$ multilayer coatings were synthesized by sequentially using sputtering and PLD. The $\mathrm{TiO}_{2} / \mathrm{PI}$ ratio was kept at 10:1. Four groups of multilayer coatings with varying periodic thicknesses (in nm) of $\mathrm{TiO}_{2} / \mathrm{PI}$ were fabricated: 100/10, $75 / 7.5,100 / 10$ with a mesolayer, $75 / 7.5$ with a mesolayer, and $50 / 5$ with a mesolayer (Fig. 1). The total thickness of the multilayer films was approximately $1000 \mathrm{~nm}$. In addition, titania and polyimide monolayer films were fabricated by sequentially manipulating PVD and PLD. The mesolayers were fabricated by cooperatively using PVD and PLD with suitable parameters. Table 1 shows the conditions and parameters used in this study.

The phases were identified by a grazing incidence X-ray diffractometer (GIXRD, TTRAX III, Rigaku, Japan) using a Cu-Ka radiation operated at $50 \mathrm{kV}$ and $300 \mathrm{~mA}$. 

LBL hybrid coatings
Table 1 Design of nacre-like

\begin{tabular}{|c|c|c|c|c|c|c|}
\hline \multirow[t]{2}{*}{ Code } & \multicolumn{3}{|c|}{ Thickness (nm) } & \multirow{2}{*}{$\begin{array}{l}\mathrm{TiO}_{2} / \mathrm{PI} \text { ratio in } \\
\text { the mesolayer }\end{array}$} & \multirow[t]{2}{*}{ Repetition } & \multirow[t]{2}{*}{ Total Thickness (nm) } \\
\hline & $\mathrm{TiO}_{2}$ & Mesolayer & PI & & & \\
\hline TPI 100 & 100 & - & 10 & - & 10 & Approx. $1000 \mathrm{~nm}$ \\
\hline TPI 75 & 75 & - & 7.5 & - & 12 & Approx. $1000 \mathrm{~nm}$ \\
\hline TPI 100 Mso & 100 & 1.5 & 7 & $5 / 1$ & 10 & Approx. $1000 \mathrm{~nm}$ \\
\hline TPI 75 Mso & 75 & 1.5 & 4.5 & $5 / 1$ & 12 & Approx. $1000 \mathrm{~nm}$ \\
\hline TPI 50 Mso & 50 & 1.5 & 2 & $5 / 1$ & 18 & Approx. $1000 \mathrm{~nm}$ \\
\hline
\end{tabular}

Fig. 1 Schematic diagram of the layer structure (TPI 50 Mso) for mesolayer insertion

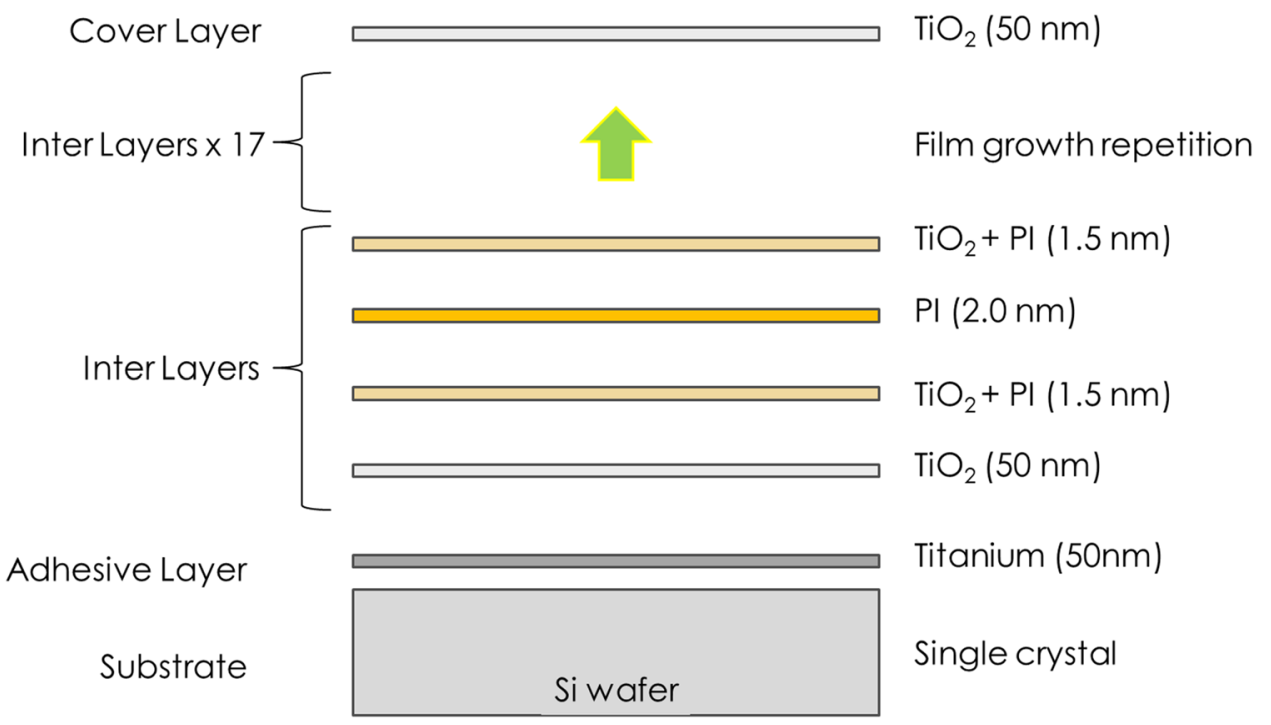

The chemical composition of the thin film was analyzed by a field emission electron probe micro-analyzer. (FEEPMA, JXA-8500F, JOEL, Japan). The morphology and microstructure were observed by field-emission SEM (JSM-7600F. JEOL, Japan). The intrinsic mechanical properties of the coatings were measured by a nanoindenter (NHT, CSM instrument) under a $5-\mathrm{mN}$ maximum test load. A loading of $100 \mathrm{mN}$ was applied to induce cracks to determine fracture toughness. Moreover, the adhesive strength was evaluated by a scratch test in a scratch mode. All TEM samples were prepared by a focused ion beam system (FIB, Helios Nanolab 600i, The FEI Company, USA). The microstructure of the multilayered structure was observed by high-resolution transmission electron microscopy (HR-TEM, JEM-3000, JEOL, Japan).

\section{Results}

Based on the architectural design shown in Table 1, two groups of comparable results can be identified. The one is conventional $\mathrm{LBL}$ coatings stacked by $\mathrm{TiO}_{2}$ and $\mathrm{PI}$ sequentially by physical deposition methods, and the other one is according to the original LBL coatings and insert mesolayers that were implied between $\mathrm{PI}$ and $\mathrm{TiO}_{2}$ interfaces. To understand the effects of repetitions and mesolayer insertions, mechanical properties were evaluated because they are the most important indices.

The results of hardness and elastic modulus are shown in Fig. 2. Hardness increased with additional repetitions, even without the insertion of mesolayers. In contrast, modulus only increased with an increase in the number of normal LBL hybrid coatings, yet decreased with an increase in the number of coatings with mesolayers in between the $\mathrm{TiO}_{2} / \mathrm{PI}$ interface. This means that the energy was transferred much farther and more layers were linked to transform the brittle tendency to extendable one. This indicates that the modified hybrid coatings can withstand more energy, i.e., the coatings became stronger and more flexible, which helps to reinforce the structural stability. This shows that the LBL structure exhibits stronger architectural performance than the two raw materials individually.

Figure 3 shows the calculated values of $H / E$ (i.e., the combined index of hardness and modulus). Compared to the studies of Burghard et al. [6, 7], a TiO 2 /PE LBL system with the same ratio of $100 \mathrm{~nm} / 10 \mathrm{~nm}$ (TPE 100) was also analyzed. In the beginning, the $\mathrm{H} / \mathrm{E}$ ratio of $\mathrm{TiO}_{2} /$ $\mathrm{PI}$ exhibited poor performance, even weaker than the original $\mathrm{TiO}_{2}$ coating. Unlike the results of TPE 100, the 
Fig. 2 Hardness and elastic modulus of LBL hybrid coatings measured by nanoindentation
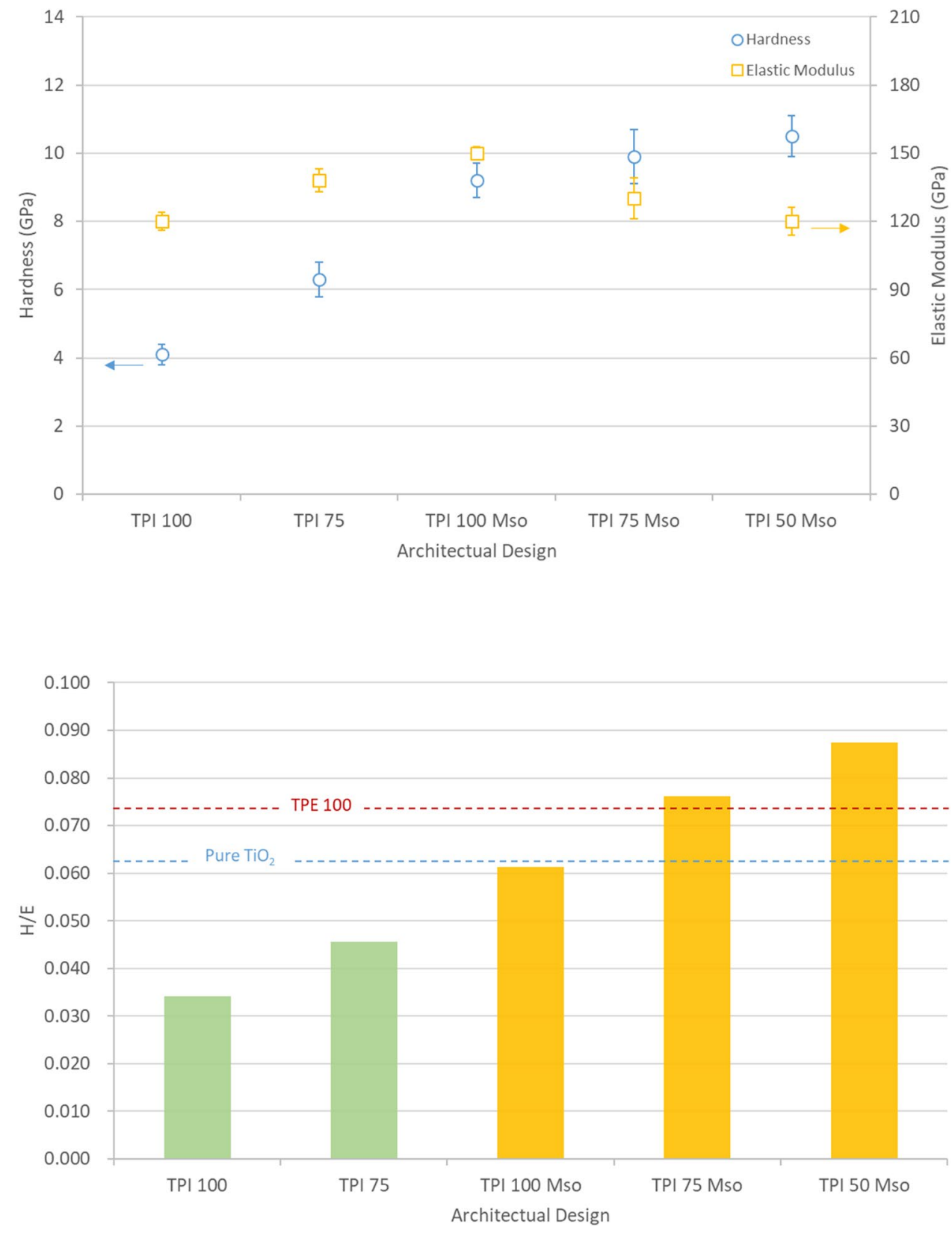

Fig. $3 H / E$ ratio of LBL hybrid coatings reinforced $L B L$ structures of the $\mathrm{TiO}_{2} / \mathrm{PE}$ system are achieved by CBD. However, the $H / E$ ratio increased with the insertion of mesolayers into the TPI system. The H/E ratio of TPI 100 Mso was approximately equal to that of the original $\mathrm{TiO}_{2}$ coating and surpassed that of TPE 100 when the thickness of each interlayer decreased and the number of layers increased. The H/E value of TPI 50 Mso was approximately $15-17 \%$ higher than that of the TPE 100 hybrid coating system.

To characterize the improvement in adhesion between $\mathrm{TiO}_{2}$ and PI, fracture toughness was also measured and calculated by:

$$
K_{1 C}=\alpha\left(\frac{E}{H}\right)^{1 / 2}\left(\frac{P}{C^{3 / 2}}\right)
$$

By calculating fracture toughness, it was determined that the values of the LBL coating with mesolayer insertions were higher than those of the original LBL coating and pure $\mathrm{TiO}_{2}$ and $\mathrm{PI}$ coatings.

Figure 4 shows the trends compared to $H / E$ values. Fractural toughness improved by periodically decreasing the thickness and inserting mesolayers. The $K_{1 c}$ value considerably increased when the mesolayers were added, and the value was higher than that of the $\mathrm{TiO}_{2} / \mathrm{PE}$ system 
Fig. 4 Calculated fractural toughness of LBL coatings compared with those of pure $\mathrm{TiO}_{2}$ and TPE 100

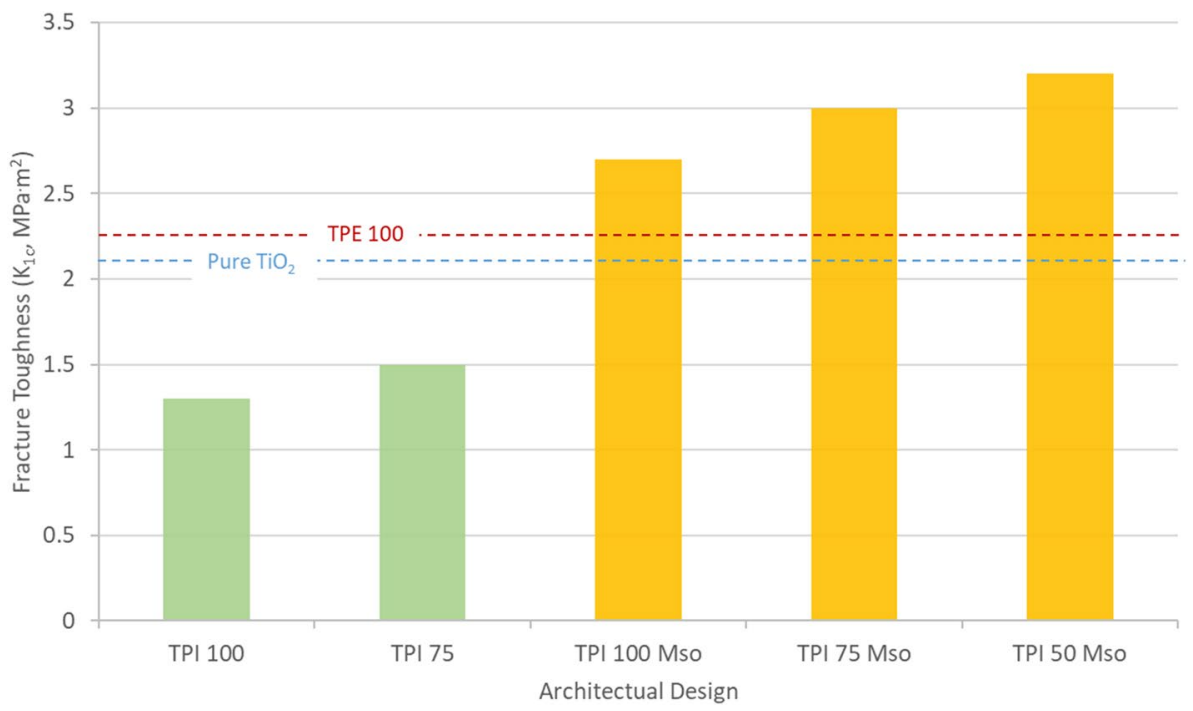

$[6,7]$. Specifically, TPI 100 Mso and TPI 75 Mso were twice as strong as the original TPI 100 and TPI 75, respectively. In addition, the energy absorbability of TPI 50 Mso was greater than that of TPE 100 by approximately $40 \%$.

By performing a corresponding scratch test, it was determined that the propagated cracks were suppressed and a greater adhesion was achieved between $\mathrm{TiO}_{2}$ and $\mathrm{PI}$. Figure 5 a further confirms the abovementioned observation. Before the indentation tip penetrated into LBL coatings, unwanted cracks were revealed. It is argued that the brittle ceramics (i.e., $\mathrm{TiO}_{2}$ ) cannot absorb too much energy without the assistance from the soft material, PI. It appears that $\mathrm{TiO}_{2}$ did not adhere well to $\mathrm{PI}$ and behaved as a single material. A comparatively stronger scheme is shown in Fig. 5b. Specifically, compressive force can stop the propagation of cracks, and no significant cracks appear during the scratch test. Even when the tip touched the surface of the Si wafer, the coating remained intact. Nevertheless, LBL coatings can store more energy with an increase in the number of layers with the same total thickness without inserting mesolayer coatings, i.e., fracture toughness can be slightly increased. After inserting mesolayers as bridges to connect $\mathrm{TiO}_{2}$ and $\mathrm{PI}$, we can improve the adhesion between $\mathrm{TiO}_{2}$ and $\mathrm{PI}$ (Figs. 4 and 5). The quantitative value of adhesion can be determined by calculating crack propagation resistance (CPR) to determine the effect of mesolayer insertions using the equation shown below:

CPRs $=\mathrm{LC} 1(\mathrm{LC} 2-\mathrm{LC} 1)$

The calculated values are shown in Fig. 6. The resistance values were lower than those of pure PI and $\mathrm{TiO}_{2}$ without mesolayers. Thus, the combination of harder material and softer material to produce a new
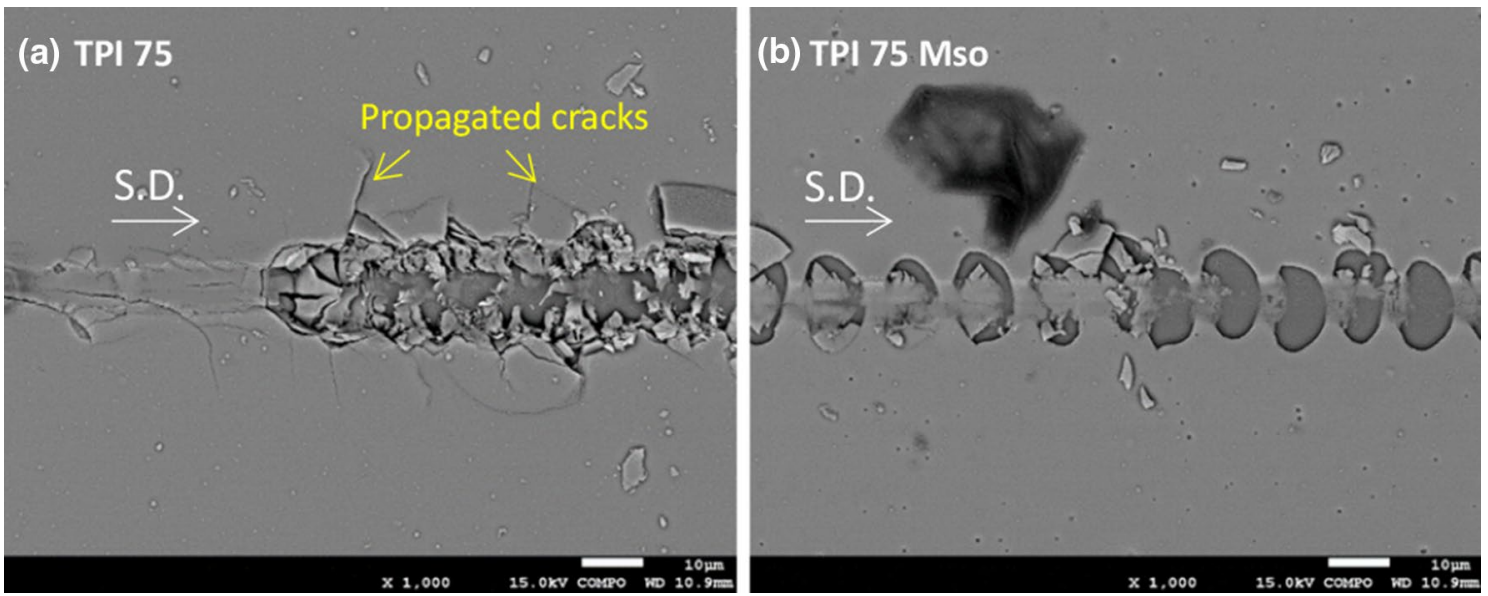

Fig. 5 SEM observation of scratch tests for $\mathbf{a}$ TPI 75 and $\mathbf{b}$ TPI 75 Mso 
Fig. 6 Calculated crack propagation resistance of $\mathrm{LBL}$ coatings compared with those of pure $\mathrm{TiO}_{2}$ and TPE 100

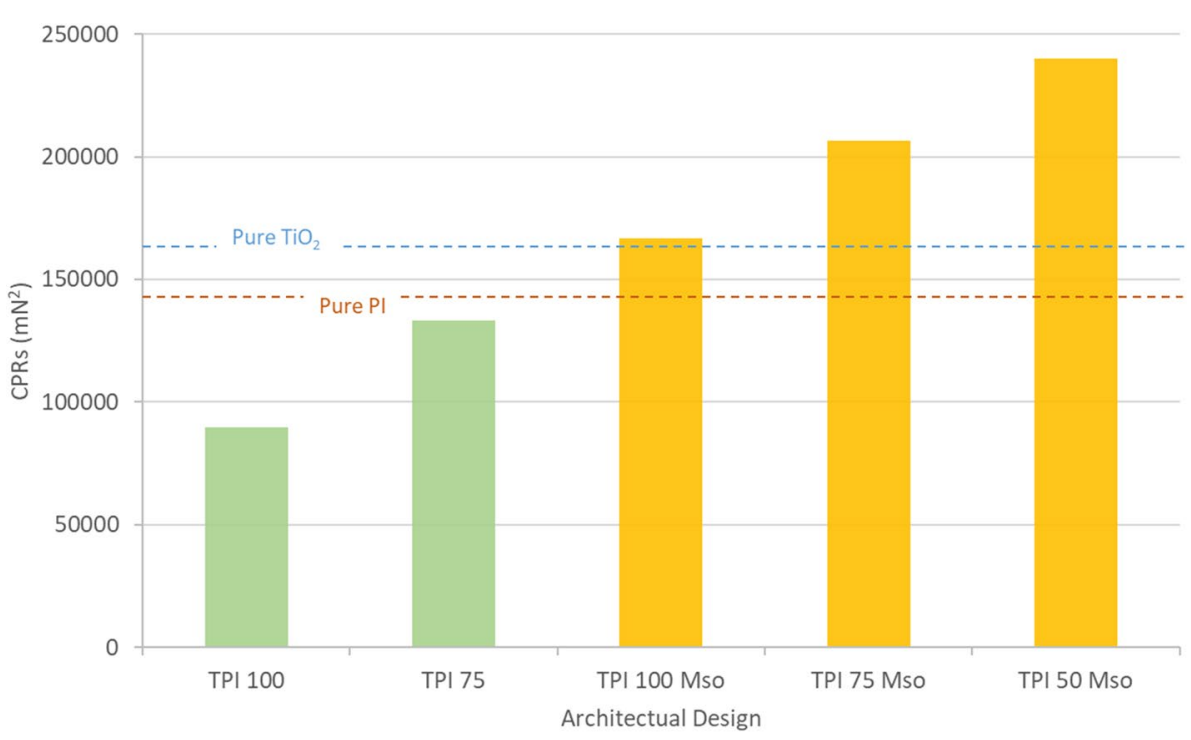

composited LBL coating did not exhibit a positive phenomenon. Subsequent to inserting a mesolayer between $\mathrm{TiO}_{2}$ and PI by sputtering and PLD co-deposition, the system could withstand and absorb more energy owing to the contribution of mesolayers, which bonded these two materials by weak bonding. To explore the created bridges in detail, HR-TEM observations were performed. Figure 7 clearly shows that the
Fig. 7 HR-TEM observation of $\mathrm{PI} /$ Meso structures for a TPI 100 and $\mathbf{b}$ TPI 50 Mso
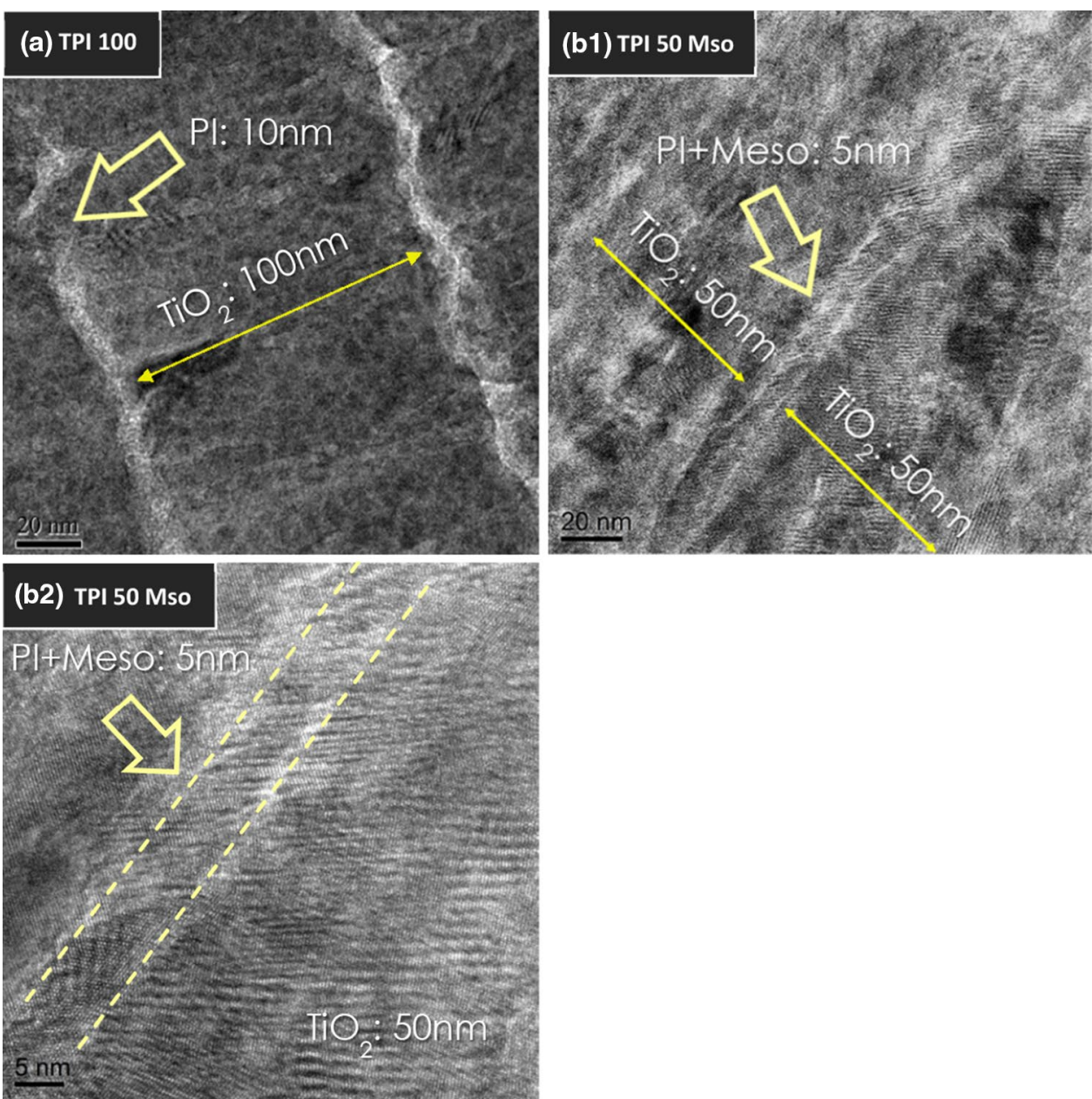
structure was accurately controlled by the designed parameters. By comparing Fig. 7a and Fig. 7b1, it is determined that the original $\mathrm{Pl}$ acted as a wall that separated $\mathrm{TiO}_{2}$ into two parts. Unlike the $\mathrm{PI}+$ meso structure, there were two meshes or small webs that linked and connected $\mathrm{TiO}_{2}$ to form a transition. Moreover, there were no significant sharp zones in the transition areas shown in Fig. 7b2. The transition areas were formed and provided a smooth crossover path for energy dissipation and additional bonding between inorganic and organic parts. In this study, two promising materials were chosen to form an LBL coating inspired by nacre. However, favorable results were not obtained using a structure similar to nacre if the two materials did not have sufficient bonding. Thus, mesolayers were added to force $\mathrm{TiO}_{2}$ and $\mathrm{PI}$ to bond by web-like interconnections. The best results were obtained by depositing more layers and inserting mesolayers between these layers, i.e., TPI 50 Mso. This structure can absorb approximately $50 \%$ more energy than that of pure $\mathrm{TiO}_{2}$ coating, and the ability to prevent cracks is $70 \%$ better than that of pure PI. The H/E ratio, fracture toughness, and CPRs values reach or surpass those of TPE $100[6,7]$, as demonstrated by TPI 75 Mso and TPI 50 Mso.

\section{Discussion}

As seen in Fig. 2, the hardness of the system elevates as a result of more repetition, whereas the elastic modulus only increases with repeated additions of conventional LBL coatings via physical deposition methods. On the other hand, the elastic modulus decreases with more repetitions during the insertion of mesolayers into the $\mathrm{TiO}_{2}$ and PI interfaces. This means that a toughened film can be mimicked since the strain that is exerted increased when stress is applied; in other words, deformation of the system becomes larger before the appropriate yield stress level is reached. The benefit of this is that, in the event of subsequent system failure, the LBL coatings are less brittle when compared to their original mechanical performance. In Fig. 8a1, it could be seen that the LBL coatings delaminated from the interfaces between $\mathrm{TiO}_{2}$ and $\mathrm{PI}$ after bending. The TEM samples shown in Figs. 8a2, b2 were prepared using focus ion beam methodology (FIB). The stress applied during experimentation procedures was very small and, thus, could be ignored. Whereas evidence of the delamination phenomena could be clearly observed in the TEM images (Fig. 8a2), there was no delamination to be seen during insertion of the mesolayers (Fig. 8b1, b2). The results from our experiments are, thus, concluded as the $H / E$ ratio, which is used to compare the combined
Fig. 8 A comparison of SEM images for a1 the original $\mathrm{LBL} \mathrm{TiO}_{2} / \mathrm{PI}$ coatings (TPI 100) and $\mathbf{b} 1 \mathrm{LBL} \mathrm{TiO}_{2} / \mathrm{PI}$ coatings with the mesolayers inserted (TPI 100 Mso). TEM images for a2 the original $\mathrm{LBL} \mathrm{TiO}_{2} / \mathrm{PI}$ coatings (TPI 100) and b2 the $\mathrm{LBL} \mathrm{TiO}_{2} / \mathrm{PI}$ coatings with the mesolayers inserted (TPI 100 Mso)
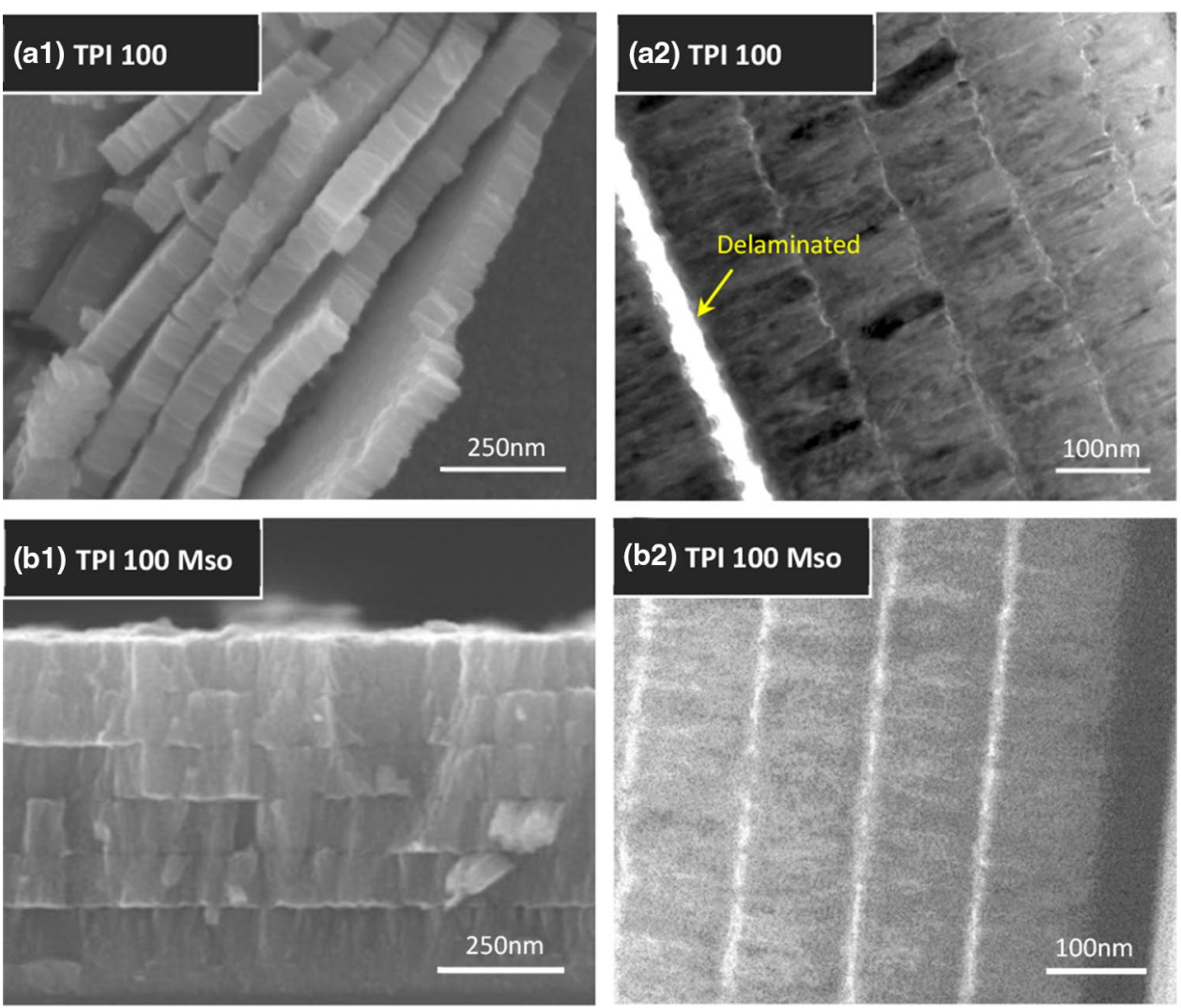

SN Applied Sciences A SPRINGER NATURE journal 
effects of hardness and elastic modulus (Fig. 3). The H/E ratio reflects the mechanical properties of the system and highlighted the novelty of the LBL coatings during mesolayer insertion. The TPI 100 Mso values for the hardest sample from the raw materials investigated in our study are similar to those of the original $\mathrm{TiO}_{2}$ coatings and can be further increased with more repetition (TPI 75 Mso and TPI 50 Mso). Finally, the values obtained for the LBL coatings that have been applied via physical processes with the respective mesolayers inserted (TPI 50 Mso) surpassed those for the $\mathrm{TiO}_{2} / \mathrm{PE}$ system that had been prepared via CBD (TPE 100).

To prove that the LBL coatings with mesolayers can be subjected to much higher energy input, the $\mathrm{TiO}_{2} / \mathrm{PI}$ interfaces are first reinforced; during this process, it is revealed that the coatings exhibited better adhesion (Fig. 8). Next, quantitative tests are conducted via the scratch test and the nanoindentation methods. From Fig. 5 a, it can be seen that extensive cracks propagated from the edges of the scar radially and perpendicular to the scratch's direction. This highlights the inherent weakness of the LBL coating since bonding between the interfaces is insufficient and cannot stop the formation of cracks. Similar results are also seen in Figs. 4 and 6. Indices related to the coating's ability to stop the propagation of cracks can be identified using the CPRs and $\mathrm{K}_{1 \mathrm{c}}$ values. If adhesion of the interfaces is poor, then the cracks' tip can propagate and spread through interfaces with lower barriers, thus dissipating the energy input that has been applied to the system. Since both the soft and tough materials, i.e., the organic portions (PI), cannot act as a means of stopping the propagation of cracks during the application of energy to the brittle portion of the system, i.e., the inorganic part $\left(\mathrm{TiO}_{2}\right)$, the path taken by the cracks extended from the surface of $\mathrm{TiO}_{2}$ and the $\mathrm{TiO}_{2} / \mathrm{PI}$ interfaces. In contrast, Fig. $5 \mathrm{~b}$ ) shows that the $\mathrm{PI}$ portion hinders the propagation of cracks, since the path of propagation does not appear on the surface and a closed-loop oval can be seen surrounding each scratch. As exemplified by the results shown in Figs. 4 and 6, the process of propagation is constrained and can be subjected to higher energy input.

It is also worth noting how the type of material used in our experiments influenced the outcome. $\mathrm{PI}$ is a thermosetting plastic, whereas $\mathrm{PE}$ is a thermoplastic polymer.
Generally, thermosetting plastic materials exhibit sturdier mechanical properties and better thermal stability. The H/E values for $\mathrm{PI}$ shown in Table 2 demonstrate that $\mathrm{PI}$ exerted more influence on the toughness of the LBL coatings. Comparing the physical properties of both $\mathrm{PI}$ and $\mathrm{PE}$, it can be seen that PI has a significantly higher melting point which translates to the preservation of its function even at higher temperatures. Thus, in light of these add benefits, i.e., the $H / E$ value and higher stability, more modern systems that mimic LBL films can be achieved via physical sputtering and PLD methods. Unfortunately, the results of the mechanical tests conducted are unexpected and show the presence of delamination. In the SEM images (Fig. 8a), poor adhesion is noted to be the root cause of these terrible performances. As shown in Fig. 6, after the introduction of mesolayers mixed with $\mathrm{TiO}_{2}$ and $\mathrm{PI}$ in the proper ratio and insertion into the interfaces between $\mathrm{TiO}_{2}$ and $\mathrm{PI}$, the CPRs values are compatible with TPE 100 in same architectural design (TPI $100 \mathrm{Mso}$ ). Furthermore, the fractural toughness of TPI 100 Mso is higher than that seen in the TPE system. This is quite an important advancement in creating the appropriate bonding interactions between $\mathrm{TiO}_{2}$ and $\mathrm{PI}$.

In Fig. 9, the schematic model that is derived by carefully observing the TEM images (Fig. 7b1) is presented. Herein, $\mathrm{Pl}$ is shown to be stretched inside the mesolayers that are cross-linked to form a web-like interlace which helps with adhesion of the PI layers, while the mesolayer still maintains the characteristics of $\mathrm{TiO}_{2}$ bonded with the pure $\mathrm{TiO}_{2}$ layer. The mesolayer, as a transient zone, is connected with $\mathrm{TiO}_{2}$ and $\mathrm{PI}$ together using physical deposition methods to insert the $\mathrm{TiO}_{2} / \mathrm{Pl}$ interfaces in the correct ratio in order to simulate natural growth mechanisms.

\section{Conclusion}

By inserting mesolayers into original coatings of the $\mathrm{TiO}_{2}$ and PI LBL architectural design, we solved the problem of poor adhesion between $\mathrm{TiO}_{2}$ and $\mathrm{PI}$. Mixed composites can be created by simultaneously performing sputtering and LPD using a lower sputtering rate and a higher LPD rate to achieve desired compositions. In this study, the mechanical performance of the produced films can exceed those
Table 2 Comparison of the candidate materials

\begin{tabular}{|c|c|c|c|c|c|c|}
\hline \multirow[t]{2}{*}{ Materials } & \multicolumn{3}{|c|}{ Mechanical properties } & \multicolumn{3}{|l|}{ Physical properties } \\
\hline & Hardness (GPa) & $\begin{array}{l}\text { Elastic mod- } \\
\text { ule (GPa) }\end{array}$ & $H / E$ & Cross-linking type & $\begin{array}{l}\text { Melting } \\
\text { point }\left({ }^{\circ} \mathrm{C}\right)\end{array}$ & CTE (ppm) \\
\hline $\mathrm{TiO}_{2}$ & 1.72 & 27.7 & 0.062 & NA & 1843 & 10 \\
\hline PE & 0.45 & 5.0 & 0.090 & Thermoplastic & 115 & 110 \\
\hline PI & 0.25 & 2.2 & 0.113 & Thermosetting & 375 & 90 \\
\hline
\end{tabular}


(a) $\mathrm{LBL} \mathrm{TiO}_{2}$ coatings

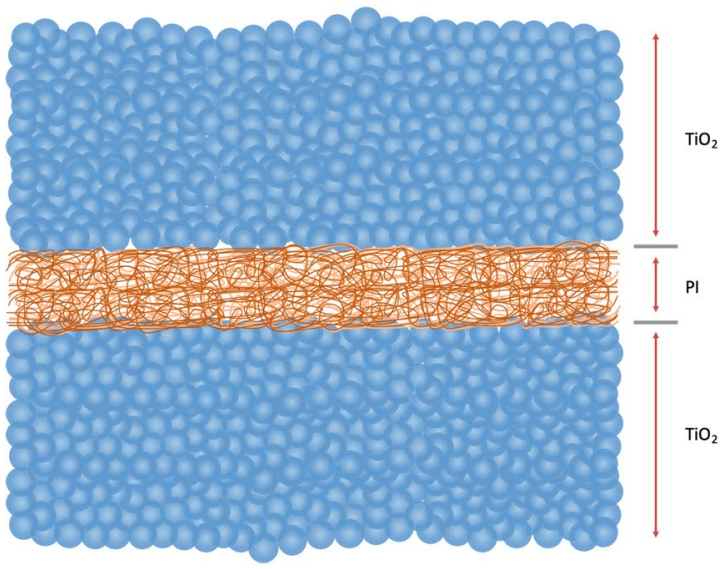

(b) $\mathrm{LBL} \mathrm{TiO}_{2}$ coatings w/ mesolayer

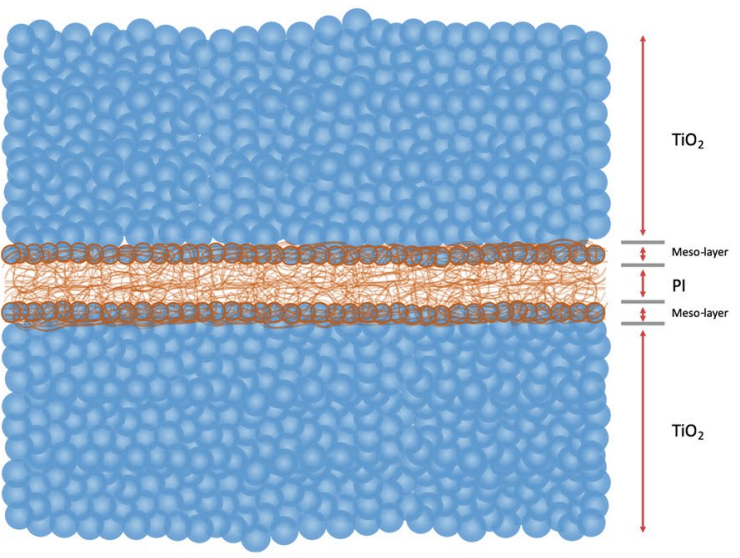

Fig. 9 Schematic mechanism for showing the correlation between the mesolayer/PI and mesolayer/TiO ${ }_{2}$ interfaces

of the original individual coatings and $\mathrm{TiO}_{2} / \mathrm{PE}$ system by increasing the number of layers. By adding mesolayers and increasing the number of layers with the same thickness, LBL coatings can be produced regardless of the chemical compatibility of materials. This approach mimics nature. Specifically, with the seasonal fluctuations in temperature, the creatures respond by forming a composite material in the transition stage. Thus, using a similar approach, a composite material (i.e., mesolayer) is created to mimic nature.

Funding This study was funded by the High Entropy Research Center of National Tsing Hua University under Project No. 107-301-F-003, and the Ministry of Science and Technology of Taiwan under Project No. 105-2221-E-007-027-MY3.

\section{Compliance with ethical standards}

Conflict of interest The authors declare that they have no conflict of interest.

\section{References}

1. Sanches C, Arribart H, Guille MMG (2005) Biomimetism and bioinspiration as tools for the design of innovative materials and systems. Nat Mater 4:277

2. Mayer $\mathrm{G}$ (2005) Rigid biological systems as models for synthetic composites. Science 310:1144

3. Ritchie RO (2011) The conflicts between strength and toughness. Nat Mater 10:817

4. Aksay IA, Trau M, Manne S, Honma I, Yao N, Zhoe L, Fenter P, Eisenberger PM, Gruner SM (1996) Biomimetic pathway for assembling inorganic films. Science 273:892

5. Sellinger A, Weiss PM, Nguyen A, Lu Y, Assink RA, Gong W, Brinker CJ (1998) Continuous self-assembly of organic-inorganic nanocomposite coatings that mimic nacre. Nature 394:256

6. Burghard Z, Tucic A, Jeurgens LPH, Hoffmann RC, Bill J, Aldinger F (2007) Nanomechanical properties of bioinspired organicinorganic composite films. Adv Mater 19:970
7. Burghard Z, Zini L, Srot V, Bellina P, Aken PAV, Bill J (2009) Toughening through nature-adapted nanoscale design. Nano Lett 9:4103

8. Batool S, Gill R, Arshad M, Siddiqi HM, Qureshi SS (2018) Layerby-layer fabrication of nacre-inspired expoxy/MMT multilayered composites. J Appl Polym Sci 135:46079

9. Chen T, Shi P, Zhang J, Li Y, Tian X, Lian J, Duan T (2018) Bioinspired enhancement of chitosan nanocomposite films via MgAcc crystallization, their robust, hydrophobic and biocompatible. Appl Surf Sci 459:129

10. Munch E, Launey ME, Alsem DH, Saiz E, Tomsia AP, Ritchie RO (2008) Tough, bio-inspired hybrid materials. Science 322:1516

11. Shi G, Wang H, Liu X, Gao C, We Z (2019) Fabrication and characterization of nacre-inspired alumina-epoxy composites. Ceram Int 45:14464

12. Mao HR, Shen P, Liu YH, Zhao YG, Jiang QC (2018) Nacreinspired lightweight and high-strength $\mathrm{AZ91D} / \mathrm{Mg}_{2} \mathrm{~B}_{2} \mathrm{O}_{5} \mathrm{~W}$ composites prepared by ice templating and pressure-less infiltration. J Mater Sci 53:12167

13. Lee JI, Wat A, Kim J, Ryu CW, Chang HJ, Park ES, Ritchie RO (2019) Synthesis of bioinspired ice-templated bulk metallic glass-alumina composites with intertwined dendritic structure. Scr Mater 172:159

14. Wilkerson RP, Gludovatz B, Watts J, Tomsia AP, Hilmas GE, Ritchie RO (2018) A study of size effects in bioinspired, "nacrelike", metal-compliant-phase (nickel-alumina) coextruded ceramics. Acta Scr 148:147

15. Yang HM, Chan YC, Hsu TH, Chen HW, Lee JW, Duh JG, Chen PY (2015) Synthesis and characterization of nacre-inspired zirconia/polyimide multilayer coatings by a hybrid sputtering and pulsed laser deposition technique. Surf Coat Technol 284:118

16. Toiserkani $\mathrm{H}$ (2015) Polyimide/nano- $\mathrm{TiO}_{2}$ hybrid films having benzoxazole pendent groups: in situ sol-gel preparation and evaluation of properties. Prog Org Coat 88:17

17. Atabaki F, Ahmadizadegan $\mathrm{H}$ (2015) Fabrication of a new polyimide/titania $\left(\mathrm{TiO}_{2}\right)$ nanocomposite thin film by the sol-gel route. Polym Plast Technol 54:523

18. Lin AYM, Chen PY, Mayer MA (2008) The growth of nacre in the abalone shell. Acta Biomatter 4:131

19. Meyers MA, Lim CT, Li A, Hairul Nizam BR, Tan EPS, Seki Y, McKittrick J (2009) The role of organic intertile layer in abalone nacre. Mater Sci Eng C 29:2398 
20. Zhang A, Chen Y, Sulivan M, Prorol, BC (2017) The growth and mechanical properties of abalone nacre mesolayer. In: Korach et.al. (eds) Mechanics of biological systems and materials, vol. $6, \mathrm{p} 143$

21. Liu C, Du J, Xie L, Zhang R (2017) Direct observation of nacre proteins in the whole calcite by super-resolution microscopy reveals diverse occlusion patterns. Cryst Growth Des 17:1966

22. Moullac GL, Schuck L, Chabrier S, Belliard C, Lyonnard P, Broustal F, Soyez C, Saulnier D, Brahmi C, Ky CL, Beliaeff B (2018) Influence of temperature and pearl rotation on biomineralization in the pearl oyster, Pinctada margaritifera. J Exp Biol 221:1

Publisher's Note Springer Nature remains neutral with regard to jurisdictional claims in published maps and institutional affiliations. 